Sādhanā Vol. 37, Part 2, April 2012, pp. 261-279. (C) Indian Academy of Sciences

\title{
Simulation of depth of penetration during ballistic impact on thick targets using a one-dimensional discrete element model
}

\author{
RAJESH P NAIR* and C LAKSHMANA RAO \\ Applied Mechanics Department, Indian Institute of Technology Madras, \\ Chennai 600 036, India \\ e-mail: rajeshpnair@gmail.com, lakshman@iitm.ac.in
}

MS received 18 August 2010; revised 23 January 2012; accepted 16 February 2012

\begin{abstract}
One-dimensional discrete element model for the ballistic impact is used to determine the depth of penetration of a bullet on a thick target. Discrete Element Method (DEM) is a numerical tool where a continuum is modelled as a network of masses connected by normal springs. A one-dimensional discrete element model is developed to obtain the displacements and forces associated with the ballistic impact on a thick target. The depth of penetration of the penetrator into the target is calculated from these DEM results. The simulated results of depth of penetration are found to be in reasonable agreement with the simulation results of other numerical approaches that are available in the literature.
\end{abstract}

Keywords. Discrete element method; one-dimensional models; ballistic impact; thick target; depth of penetration.

\section{Introduction}

Ballistic impact is the study which deals with projectile hitting the target and observing its effects in terms of deformation and fragmentation of the target. The need to model ballistic impact condition is for several applications of impact such as nuclear and military applications, where we encounter high velocity projectiles hitting a target. Numerical modelling of the process helps to reduce the expense of doing repeated experiments and also to optimize the geometry and material properties of target and penetrator. The importance of ballistic impact study (Ben-Dor et al 2005) is to know the failure pattern of target and to find the depth of penetration (DOP) associated with the impact of a penetrator on a thick target. Simulation of the impact process is a complicated task and involves the simulation of the following steps: (a) Simulation of the nonlinear contact between the penetrator and the target. (b) Simulation of large deformation and large strain of material near the contact zone. (c) Simulation of an elastic stress wave propagation

*For correspondence 
through the penetrator and the target through the equations of motion through a continuum. (d) Simulation of energy dissipative mechanisms such as plastic deformation, damage through material separation, etc.

Each of the processes outlined above is complex and is a source of nonlinearity in the mechanical response of the target and would require detailed simulation numerically. While the procedures to simulate the nonlinear contact and material simulation would be common to any numerical procedure, there could be alternate strategies available for simulation of the equations of motion and the associated strain fields in a material.

Simulation of ballistic impact process has been done using several numerical techniques. Kurtaran et al (2003), Gailly \& Espinosa (2002) have simulated the impact process using threedimensional solid elements, where a time domain analysis was done to simulate the continuum response, in classical finite element analysis of the process. Woodward (1996) used Finite Difference Method (FDM) to simulate ballistic impact process. Tavarez \& Plesha (2007) have used a DEM to simulate the deformation and failure process for a continuum media. In this method, the continuum is idealized as a network of masses connected by springs and the response of the continuum is simulated by writing the equations of motion of each of the masses. Tavarez \& Plesha (2007) have simulated the separation of matter using DEM and claims that this method will be more effective compared to Finite Element Method (FEM) when it is necessary to simulate the separation of media during impact.

Ballistic impact on thick targets is normally associated with a crater generation by the penetrator, where it is difficult to infer the existence of any separation. An estimation of DOP associated with this process can also be done using any of the numerical procedures outlined above. In addition, the associated equations can also be solved by a simple finite difference technique and this has been done by Woodward (1996). In our study, the focus is on the simulation of DOP associated with a thick target, using the DEM. In future, the DEM procedure that is established here will be used to simulate complicated processes such as penetration in thin targets (where there is a possibility of separation) and two-dimensional impact processes.

For problems with severe damage, DEM offers a number of attractive features over continuum based numerical methods, with primary feature being a seamless transition from solid phase to particulate phase. Continuum based methods such as FEM are challenging to apply to these problems and are plagued by the need for continuum constitutive models, severe element distortion and frequent re-meshing. DEM by Magnier \& Donze (1998) is also a recent numerical technique that has been used to predict the response of a continuum in several applications including impact.

Cundall \& Strack (1979) originally proposed DEM and it is a numerical technique for evaluation of stresses and displacement in a continuum. In this method, each element is treated as an independent rigid mass which interacts with other similar masses through deformable springs that exist at the inter element interface. In DEM, equations of motion are written for each discrete mass and these equations are solved using an explicit numerical scheme. The DEM (Sitharam 2000) has been successfully used to simulate a number of deformational processes such as the compression test, three point bend test on concrete, footing operation, progressive caving of mine simulation, etc. In literature it is found that inelastic rock impact using DEM is carried out by An \& Tannant (2007). Raje et al (2007) successfully used rigid discrete elements to model material continuum for evaluating the stress in bearing contacts. Prochazka (2004) introduced discrete hexagonal element for simulating fracture process in rock in 2-D domain.

Numerical modelling of ballistic impact analysis is possible using one-dimensional, twodimensional or in three-dimensional models of material deformation and failure. While the true response of any impact process is a three-dimensional in nature, simulation of a three-dimensional is 
too complex and is not amenable for developing an understanding into the impact phenomenon, or to study the response through a parametric study. In 3-D modelling the mesh size dependency on the result is more and also the total time for simulation is much higher compared to one-dimension modelling. The advantage of one-dimensional model is that there is less number of parameters in the mathematical formulation and hence it is easy for us to obtain first order estimates of design parameters such as DOP, residual velocity (velocity of penetrator coming out from target after full penetration), etc. with reasonable accuracy. The disadvantage of onedimensional modelling is that the multi-dimensional stress distributions cannot be obtained in the simulation.

In ballistic impact modelling of penetrator and target, one-dimensional analytical models as well as numerical models are available. These models can be used to make an estimate of the ballistic parameters. Forrestal et al (1995) developed one-dimensional analytical model for thick target in which failure pattern is predicted based on spherical cavity expansion. The assumption used is that radial velocity produced by penetrator for making cavity in target is equal to the particle velocity in the target at the nose target interface. A closed form equation for the DOP due to rigid spherical nose rods that penetrate ductile metal targets using the Newton's second law of motion for the entire body, treating it as a rigid mass, is obtained (Forrestal et al 1995). Awerbuch \& Bodner (1974) developed a mathematical model to describe the mechanics of normal perforation (complete penetration) of projectile into metal targets. This model seems capable of predicting residual velocity of projectile, contact time and force time histories. But this model is applicable only for thin metal targets where the projectile will come out from the non-impact end of the target after penetration process. Anderson et al (1993) used energy balance principle and used numerical simulations to examine long rod penetration as a function of impact velocity. Ravid et al (1987) highlighted resistance to DOP based on momentum balance principle.

The numerical model proposed by Woodward (1996) based on FDM is used for ballistic impact study of penetrator on thick targets. The deformation of target and penetrator was studied by considering different strength for target and penetrator (Woodward 1996). The development of one-dimensional numerical model is done in Woodward's (1996) paper by considering the projectile and target as cylinders. Numerical Results (FDM) are compared with experimental results. The limitations of using a difference equation to model a differential equation in space, which is a limitation of any FDM, will continue to exist in the method adopted by Woodward (1996).

In our present work, we are simulating ballistic impact process using Discrete Element Method in a one-dimensional domain (1-D DEM). DEM was chosen as the numerical procedure, to explore the promise it holds in modelling material separation. A one-dimensional model is used in the current work because of its simplicity and also possible adequacy to get a first order estimate of the DOP. The paper is organized in the following way. Introduction and literature review was explained in section 1. The basic steps in DEM are given in section 2. Adaptation of DEM for ballistic impact is explained in section 3. Section 4 shows the results obtained in 1D DEM for ballistic impact simulation and the comparison of results with 1-D FDM and experimental results. Conclusions about the results obtained in simulation are discussed in section 5 .

\section{Basic steps in DEM}

Cundall \& Strack (1979) have introduced the DEM as a numerical technique which can be applied to discontinuous bodies like granular materials. Discrete numerical model are used 


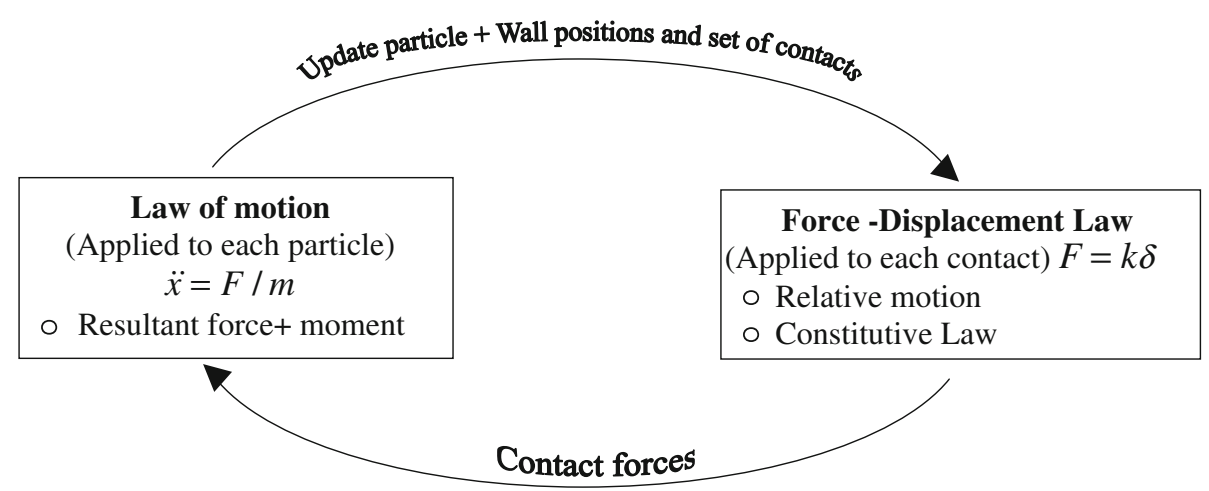

Figure 1. Principle of DEM.

in Cundall \& Strack (1979) paper for granular assemblies which is capable of describing the mechanical behaviour assemblies of discrete elements which are either circular discs or spheres. In DEM time-step chosen is so small that during a single time step, disturbances will not propagate from one discrete element to its immediate neighbouring element. It is this advantage of DEM which makes it possible to model the interaction of large number of elements without requiring high memory capacity. The total number of time steps taken for DEM simulation is in such a way that contact force remains same even if we increase the number of time steps. Two laws are basically used in DEM. They are; (i) Force- displacement law at the contact points to find contact force and (ii) Newton's second law of motion to find out displacement of each discrete element. The DEM is used to find out the contact deformation and forces at each contact points. The principle of DEM is shown in figure 1 .

2.1 Modelling the geometry of material as an assembly of discrete element particles connected by normal and shear springs

Modelling the geometry is an important part of DEM. The mass of the solid body is lumped to each discrete element which is assumed to be rigid bodies having deformation at the contact points. These deformations are taken care by the imaginary springs that exist between two discrete elements (figure 2).

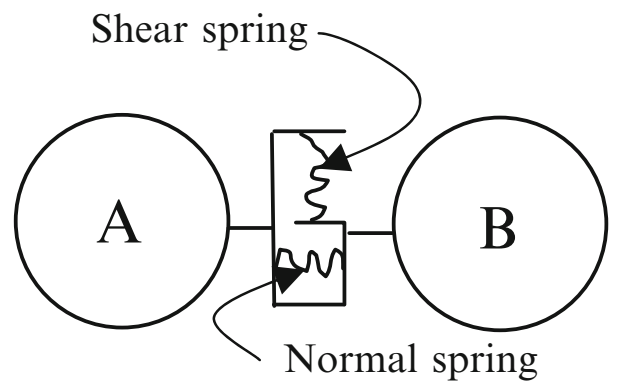

Figure 2. Contact between two discrete elements. 


\subsection{Determination of spring constants $\left(K_{n}\right.$ and $\left.K_{s}\right)$ of the connecting springs}

The stiffness at the inter element boundaries is determined by developing a discrete element model of a unit cell of material. Uniaxial stress is applied on unit cell to determine the normal and shear stiffness. This technique is used by Tavarez \& Plesha (2007) for finding out inter element stiffness of isotropic material modelled using circular two-dimensional discrete elements. The macroscopic linear elastic behaviour of isotropic materials can be characterized by two elastic constants which are Young's modulus (E) and Poisson's ratio (v). For two-dimensional discrete element modelling; the spring constants $\left(\mathrm{K}_{n}\right.$ and $\left.\mathrm{K}_{s}\right)$ are determined by uniaxial test and using linear elastic isotropic stress-strain law. Complete details of determination of inter element stiffness can be found in Tavarez's (2005) thesis.

\subsection{Imposing the boundary conditions and loading for the modelled geometry}

The boundary conditions exist at the contact points of the discrete elements. It can be either displacement boundary conditions or force boundary conditions. Boundary conditions (BC) need to be defined in DEM to avoid rigid body motions.

\subsection{Formulation of GDE with the BC's}

Unlike the equation of equilibrium in static condition, in ballistic impact which is a dynamic simulation, the equation of motion is considered as the basic Governing elasto-dynamics Differential Equation (GDE) to solve the problem. This GDE is solved using the boundary and initial conditions. The equation of motion in three-dimensions for the wave propagation in elastic medium (Timoshenko \& Goodier 1970) is represented by (1)

$$
\begin{aligned}
(\lambda+G) \frac{\partial e}{\partial x}+G \nabla^{2} u & =\rho \frac{\partial^{2} u}{\partial t^{2}} \\
(\lambda+G) \frac{\partial e}{\partial y}+G \nabla^{2} v & =\rho \frac{\partial^{2} v}{\partial t^{2}} \\
(\lambda+G) \frac{\partial e}{\partial z}+G \nabla^{2} w & =\rho \frac{\partial^{2} w}{\partial t^{2}},
\end{aligned}
$$

where $\lambda$ is the Lame's parameter, $\mathrm{G}$ is the bulk modulus, e is the volume expansion, $\rho$ is the density of the material and the symbol $\nabla^{2}$ represents the operation (2)

$$
\nabla^{2}=\frac{\partial^{2}}{\partial x^{2}}+\frac{\partial^{2}}{\partial y^{2}}+\frac{\partial^{2}}{\partial z^{2}} .
$$

The LHS of (1) represents the forces due to deformation and the RHS represents the inertia force.

\subsection{Solving the governing equations for the given boundary conditions}

The GDE are solved by converting the differential equation to difference equation using the central difference scheme (CDS). Force and displacements at the boundaries of each discrete element is calculated by using Newton's second law of motion and force-displacement law. 


\section{Adaptation of DEM for ballistic impact}

Impact condition (Stronge 2004) consists of sudden force applied within a short period of time. Stress wave propagation (Hu \& Eberhard 2004) is an important parameter in impact condition. In our paper, 1-D DEM for ballistic impact analysis is carried out by assuming (i) nonlinear analysis due to plastic deformation in the target and penetrator and (ii) no dissipation of energy due to heat generation. Considering the ballistic impact simulation using DEM in 1-D domain, all the formulations will have one variable only, which is variable in impact direction. Positive direction for contact forces is taken along the positive direction of initial velocity of penetrator.

Modelling of ballistic impact problem using 1-D approach is carried out using DEM. A flatended copper bullet (penetrator) hitting a thick 2024-T351 aluminum plate (target) is studied. The material and geometric parameters are given in table 1. Here, in the 1-D DEM simulation, the penetrator is deformable and is also allowed to penetrate through the target. The target is taken thick enough so that no perforation (complete penetration) occurs when the bullet penetrates through the target. Important parameter obtained in thick target analysis is DOP. The schematic representation of flat-ended penetrator and target is as shown in figure 3 .

Table 1. Penetrator, target and discrete elements parameters used in 1-D modelling for flat end penetrator.

\begin{tabular}{ll}
\hline Parameter & Value \\
\hline & \multicolumn{1}{c}{ Copper penetrator characteristics } \\
Length & $12.74 \mathrm{~mm}$ \\
Diameter of penetrator & $4.8 \mathrm{~mm}$ \\
Density & $8500 \mathrm{~kg} / \mathrm{m}^{3}$ \\
Poisson's ratio & 0.34 \\
Young's modulus & $114 \mathrm{GPa}$ \\
Height of a discrete element & $0.32 \mathrm{~mm}$ \\
Number of discrete element used & 40 \\
Flow stress & $443 \mathrm{MPa}$ \\
Total mass of penetrator model & $1.96 \times 10^{-3} \mathrm{Kg}$ \\
Velocity of penetrator & $230 \mathrm{~m} / \mathrm{sec}$ \\
& $2780 \mathrm{~kg} / \mathrm{m}^{3}$ \\
Density & 0.33 \\
Poisson's ratio & $70 \mathrm{GPa}$ \\
Young's modulus & $25 \mathrm{~mm}$ \\
Thickness of target & $4.8 \mathrm{~mm}$ \\
Diameter of target discrete element & $0.31 \mathrm{~mm}$ \\
Height of a discrete element & 80 \\
Number of discrete element used & $776 \mathrm{MPa}$ \\
Flow stress & $324 \mathrm{MPa}$ \\
Yield stress & $1.22 \times 10^{-3} \mathrm{Kg}$ \\
Total mass of 1-D target model & $4.85 \times 10^{-9} \mathrm{sec}$ \\
Discrete element model parameters & For velocity of impact $=230 \mathrm{~m} / \mathrm{sec})$ \\
Time step for simulation & $95 \mathrm{steps}$ \\
Total number of time steps & \\
\hline
\end{tabular}




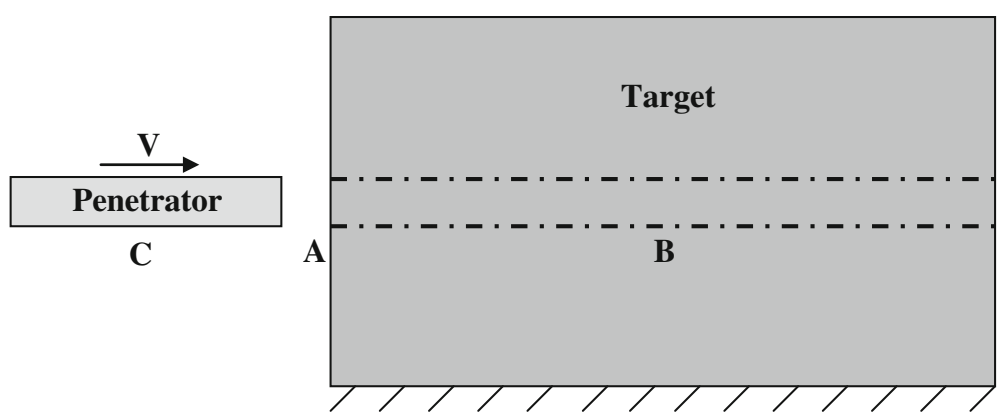

Figure 3. Schematic diagram of penetrator and target arrangement for ballistic impact.

\subsection{Modelling of ballistic impact using one-dimensional discrete elements}

The portion of target which is in line with penetrator alone is modelled in 1-D domain, since that portion alone is subjected to high compression. Ballistic impact in 1-D DEM is modelled as a rod impacting another stationary rod as shown in figure 4 using 1-D discrete bar elements. The mass of the discrete element is lumped to the points and springs are connecting the masses. The spring mass analogy for ballistic impact simulation is shown in figure 5 .

\subsection{Determination of spring constants for ballistic impact simulation}

In DEM, the stress-strain relationship in a material is converted into force-displacement relations associated with masses and springs. Uniaxial stresses and strains experienced in the rod are converted into force acting on masses and displacement in the springs. The determination of spring stiffness between the masses, from the known stress-strain relation of the rod is illustrated for the two cases of (i) linear elastic material and (ii) nonlinearly deforming material, in the following subsections.

3.2a Calculation of linear spring stiffness: The stress $\sigma$ and strains $\varepsilon$ in an elastic rod are related to each other through the Young's modulus $E$ as per the relation given below (3)

$$
\sigma=E \varepsilon
$$

The linearised strain-displacement relationship in the rod is given by

$$
\varepsilon=\frac{\Delta u}{\Delta x}
$$

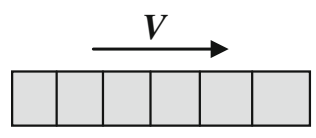

C

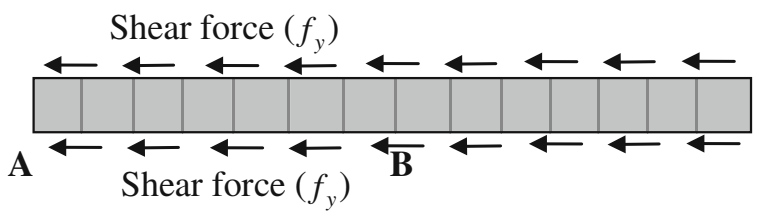

Figure 4. One-dimensional discrete element model for impact analysis incorporating shear forces on the boundaries of discrete elements of target. 


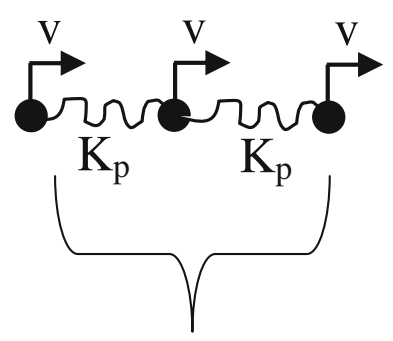

Penetrator

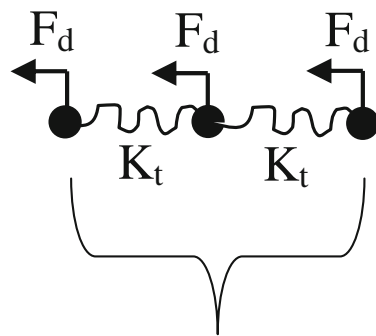

Target

Figure 5. Spring-mass analogy for penetrator and target.

Normal stress in the rod is related to the force $F$ on the discrete element mass by

$$
\sigma=\frac{F}{A},
$$

where A is the cross sectional area of the rod. (3) is rewritten using (4) and (5) as

$$
\frac{F}{A}=E \frac{\Delta u}{\Delta x} \text {. }
$$

Normal spring stiffness $\left(K_{n}\right)$ is defined as the force $F$ produced in spring per unit deformation $\Delta u$.

$$
K_{n}=\frac{F}{\Delta u}=\frac{E A}{\Delta x}=\frac{E A}{l},
$$

$\Delta x$ is equal to $l$ in 1-D DEM for ballistic impact analysis. $l$ is the length of each discrete element.

3.2b Calculation of nonlinear spring stiffness: Since ballistic impact is a nonlinear phenomenon involving plastic deformation of the materials, nonlinear spring stiffness is required to capture the deformation between discrete elements. In order to get the nonlinear spring stiffness, a power law is used. This nonlinear stress-strain relationship is taken from Woodward (1982). The nonlinear stress-strain relationship is taken as

$$
\sigma=\sigma_{0} \varepsilon^{n}
$$

where the strength parameters $\left(\sigma_{o}\right.$ and $\left.n\right)$ were obtained by performing uniaxial compression tests (Woodward 1982) on the materials and fitting the results for true stress $(\sigma)$ and true strain $(\varepsilon)$ to (8). For penetrator $\sigma_{0}$ and $\mathrm{n}$ are $\sigma_{p}$ and $\mathrm{n}_{p}$ with the values of $443 \mathrm{MPa}$ and 0.1 , respectively. For target $\sigma_{0}$ and $\mathrm{n}$ are $\sigma_{t}$ and $\mathrm{n}_{t}$ with the values of $776 \mathrm{MPa}$ and 0.096 , respectively. Using the strain-displacement and force-displacement relation; the spring stiffness is derived. As predicted by Woodward (1982), for target the stress-strain relationship is

$$
\sigma=2.7 \sigma_{t} \varepsilon^{n_{t}}
$$

The strain displacement law and force-stress relationship is

$$
\varepsilon=\frac{\Delta u}{\Delta x} \quad \text { and } \quad F=\sigma A .
$$


Thus (9) can be written as

$$
F=2.7 \sigma_{t} A \varepsilon^{n_{t}-1} \frac{\Delta u}{\Delta x},
$$

where $\Delta x=l_{t}$, thus (11) can be written as

$$
F=K_{t} \Delta u
$$

where $l_{t}$ is the length of target element and the normal spring stiffness between each target element $\left(K_{t}\right)$ is given as

$$
K_{t}=\frac{\left(2.7 \sigma_{t} A \varepsilon^{n_{t}-1}\right)}{l_{t}} .
$$

Similarly, the normal spring stiffness between each penetrator element $\left(K_{p}\right)$ is derived from (8) as

$$
K_{p}=\frac{\left(\sigma_{p} A \varepsilon^{n_{p}-1}\right)}{l_{p}} .
$$

\subsection{Boundary conditions}

For the ballistic impact simulation the displacement boundary condition alone exists. Boundary conditions for the target is

$$
\begin{aligned}
& V_{t 1, t}=V_{p 1, t}=V_{0}, \quad u_{t 1, t}=V_{0} \Delta t \text { for } \quad x=0 \text { and } t=0 \\
& V_{t n t, t}=0, \quad u_{t n t, t}=0 \quad \text { for } x=L_{t} \text { for all } t^{\prime} \text {, }
\end{aligned}
$$

where ' $x=0$ ' represents the impact end of target (point A of figure 4) and ' $x=L_{t}$ ' represents the non-impact end of target. ' $t=0$ ' represents the time at which impact occurs. $V_{t 1, t}$ is the velocity of target element (element 1 ) at the impact end, $V_{p 1, t}$ is the velocity of penetrator element (element 1 ) at the impact end, $V_{0}$ is the initial velocity of penetrator. $\Delta t$ is the time step in discrete element simulation (section 3.5) and $u_{t 1, t}$ is the displacement of the target element at the impact end. $V_{t n t, t}$ is the velocity of target element at the non-impact end, $u_{t n t, t}$ is the displacement of the target element at the non-impact end.

Boundary conditions for the penetrator is

$$
\begin{array}{ll}
V_{p 1, t}=V_{0}, \quad u_{p 1, t}=0 & \text { for } x=0, t<0 \\
V_{p 1, t}=V_{0}, \quad u_{p 1, t}=V_{0} \Delta t & \text { for } x=0, t=0 \\
V_{p 1, t}<V_{0}, \quad u_{p 1, t}=V_{p 1, t} \Delta t & \text { for } x=0, t>0,
\end{array}
$$

where ' $x=0$ ' represent the impact end of penetrator (point A of figure 4). $V_{p 1, t}$ is the velocity of penetrator element at the impact end and $u_{p 1, t}$ is the displacement of the penetrator element at the impact end. ' $t<0$ ' represents the time before impact occurs, ' $t=0$ ' represents the time at which impact occurs and ' $t>0$ ' represents the time after impact.

\subsection{Formulation of GDE with the given BC's for the ballistic impact problem}

The GDE in one-dimension is shown in (17)

$$
m\left(\frac{\partial^{2} u}{\partial^{2} t}\right)+F_{y}=\sum F .
$$


Here, RHS represents the total force acting on each discrete element, first term on LHS represents the inertia force and second term on LHS represents the shear force acting on the boundary of each discrete element.

\subsection{Solving the governing differential equation for ballistic impact simulation}

Basic forces that act in the ballistic impact simulation are inertial force, shear forces and the spring forces. If a single discrete element is analysed, the force balance equation from figure 6 is

$$
F_{I}+F_{y}=K_{n} u_{1}+K_{n} u_{2},
$$

where $F_{I}$ is the inertial force on the mass $F_{y}$ is the shear force on the boundary of target elements and $K_{n} u_{1}$ and $K_{n} u_{2}$ are the spring forces $\left(\mathrm{Fs}_{1}\right.$ and $\left.\mathrm{Fs}_{2}\right)$ acting on both sides of the target mass. Above equation can be simplified as

$$
m_{t}\left(\frac{\partial^{2} x}{\partial^{2} t}\right)+F_{y}=K_{n} u_{1}+K_{n} u_{2} .
$$

Here, $m_{t}$ is the mass of target element, $u_{1}$ and $u_{2}$ is the deformation at both ends of the target mass. The above basic GDE is solved using CDS. The differential equation is converted to difference equation and is solved as explained in the following steps.

In 1-D DEM simulation for ballistic impact, at the first time step $\left(\mathrm{t}_{1}\right)$ deformation is assumed to occur only at the impact point i.e., on the impact side of both target and penetrator discrete elements. From second time steps onwards the deformation progress to the other contact points of discrete elements inside target and penetrator elements. Thus with time increment the deformation progresses to the non-impacting end of penetrator and target.

DEM is an explicit integration scheme where time step for simulation is an important parameter to ensure numerical stability. Critical time step $\left(\Delta t_{c}\right)$ is calculated from (20) which is obtained from Bathe (1996). The time step ( $\Delta t)$ for DEM need to be less than critical time step $\left(\Delta t_{c}\right)$ in order to ensure stability of result. Here 0.1 is the factor (Hart et al 1988) taken to ensure stability of numerical simulation (21).

$$
\begin{gathered}
\Delta t_{c}=\sqrt{2 \frac{m_{\min }}{K_{\max }}} \\
\Delta t=0.1 \sqrt{2 \frac{m_{\min }}{K_{\max }}},
\end{gathered}
$$

where $m_{\min }$ is the minimum mass of the element used in DEM and $K_{\max }$ is the maximum contact stiffness of the discrete element.

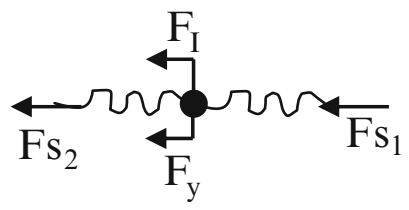

Figure 6. Forces acting on a single target discrete element mass. 
Cundall \& Strack (1979) first reported the use of DEM for analysing granular materials. In ballistic impact simulation (22), (23) and (25) are the same equations used by Cundall \& Strack (1979) for analysing the compression of two discs between rigid walls. The contact force at impact end of target discrete element $\left(F_{c t 1, t}\right)$ and penetrator element $\left(F_{c p 1, t}\right)$ at time $\mathrm{t}_{1}=\Delta \mathrm{t}$ is obtained from force displacement law as shown in (22).

where

$$
\begin{aligned}
& F_{c t 1,1}=K u_{t 1,1} \\
& F_{c p 1,1}=-K u_{t 1,1},
\end{aligned}
$$

$$
K=\frac{K_{p} K_{t}}{\left(K_{p}+K_{t}\right)} .
$$

The effective spring stiffness $K$ is the combination of both penetrator and target springs in series connection. The calculation of deformation at the impact point $u_{t 1,1}$ is given in (26). The impact force is equal to contact force at the impact end, which will be equal for both target $\left(F_{c t 1, t}\right)$ and penetrator element $\left(F_{c p 1, t}\right)$.

$F_{c p 1,1}$ act in the opposite direction of velocity of impact. $F_{c p 1,1}$ is negative because positive direction of force is taken along the velocity of impact direction. Total force on the discrete element of target $\left(F_{t 1, t}\right)$ and penetrator $\left(F_{p 1, t}\right)$ at impact end at any time will be equal to sum of all contact forces acting on the first target element and first penetrator element, respectively.

$$
\begin{aligned}
F_{p 1,1} & =F_{c p 1,1} \\
F_{t 1,1} & =F_{c t 1,1} \\
F_{p 1, t} & =F_{c p 1, t}-F_{c p 2, t}+F_{y} \\
F_{t 1, t} & =F_{c t 1, t}-F_{c t 2, t}-F_{y} .
\end{aligned}
$$

The effect of boundary is taken care by introducing shear force in the target elements which is shown in figure 4 . The shear force $\left(F_{y}\right)$ on the target element is given by

$$
F_{y}=C \pi d h \sigma_{f},
$$

where $\mathrm{C}$ is the constant calculated from distortional energy theory for failure, which predicts that the yield strength in shear is 0.577 times the yield strength in tension. Diameter of penetrator and length of target discrete element are $\mathrm{d}$ and $\mathrm{h}$, respectively. The flow stress of the target element is represented by $\sigma_{f}$ (table 1). The same (24) was used by Woodward (1982) for modelling the ballistic impact using FDM. When the penetrator enters the target, penetrator also experiences the shear force $F_{y}$, whose expression is same as (24) in which the shear force term consists of diameter of penetrator, height of penetrator element and flow stress of penetrator.

When impact occurs, velocity of target element at the impact point is taken as equal to velocity of penetrator element. By integrating the velocity with respect to time, initial displacement at the impact point is obtained (25). From the incremental time step $\Delta t$, which is obtained from (21), discrete element simulation is repeated for $n$ number of time steps till the DOP value remains constant with respect to time. The total time for simulation $T$ is the time required in 1-D DEM to obtain saturation value of DOP.

$$
\begin{aligned}
u & =\int_{0}^{T} V(t) d t \\
T & =n \Delta t .
\end{aligned}
$$


The velocity $V(t)$ is a function of time (28). For the penetrator discrete element; $V(t)$ will decrease with respect to time and will eventually become zero when the saturation value of DOP is reached. The initial deformation at the impact point is given by (26)

$$
u_{p 1,1}=u_{t 1,1}=V_{o} \Delta t
$$

The initial velocity of penetrator is represented by $V_{o}$. In the second time step the increment in deformation at the impact point for penetrator $\left(u_{p 1,2}\right)$ and target $\left(u_{t 1,2}\right)$ is calculated using CDS as shown in (27)

$$
\begin{aligned}
& u_{p 1,2}=\frac{F_{p 1,1}}{m_{p}} \Delta t^{2}+2 u_{p 1,1} \\
& u_{t 1,2}=\frac{F_{t 1,1}}{m_{t}} \Delta t^{2}+2 u_{t 1,1},
\end{aligned}
$$

where $\mathrm{m}_{p}$ and $\mathrm{m}_{t}$ are the mass of penetrator and target discrete element, respectively. Deformation in other contact points of discrete elements inside the target and penetrator is calculated from the deformation produced at the interface of two discrete elements. From the force-displacement law the contact forces are calculated and the process is repeated for every time step. In onedimensional formulation of DEM the basic equations are almost same with 1-D FDM except that the force is obtained directly from displacement in DEM. In FDM, stress is the additional parameter needed to be calculated and from stress, force is calculated. Details of 1-D FDM formulation are given in Woodward (1996).

\subsection{Calculation of depth of penetration (DOP) for ballistic impact simulation}

DOP is the depth of the hole formed on the target by penetrator after the impact. The DOP is calculated as the total distance travelled by the penetrator before its velocity comes to zero. The penetrator hits the target with an initial velocity of $V_{0}$. The numerical simulation is assumed to start from the time that the penetrator touches the target. DOP is calculated as shown in (28).

$$
\begin{aligned}
\mathrm{V}_{\mathrm{p} 1,1} & =\mathrm{V}_{0} \\
\mathrm{X}_{\mathrm{p} 1,1} & =\mathrm{V}_{\mathrm{p} 1,1} \Delta \mathrm{t} \\
\mathrm{V}_{\mathrm{p} 1,2} & =\mathrm{V}_{\mathrm{p} 1,1}-\mathrm{F}_{\mathrm{p} 1,1} \Delta t / \mathrm{m}_{\mathrm{p}} \\
\mathrm{X}_{\mathrm{p} 1,2} & =\mathrm{X}_{\mathrm{p} 1,1}+\mathrm{V}_{\mathrm{p} 1,2} \Delta \mathrm{t} \\
\mathrm{P} & =\mathrm{X}_{\mathrm{p} 1, \mathrm{~T}},
\end{aligned}
$$

$\mathrm{V}_{p 1,1}$ is the velocity of 1 st penetrator element at first time step. $\mathrm{X}_{p 1,1}$ is the distance travelled by 1 st penetrator element after the first time step. DOP of penetrator $(\mathrm{P})$ is equal to $\mathrm{X}_{p 1, T}$. In 1-D DEM; it is possible to find out the contact deformation, contact force, velocity and distance travelled by each discrete element (penetrator/target) with respect to time using (28).

\section{Ballistic impact simulation results using 1-D DEM}

MATLAB 7.0 platform is used to simulate impact process using 1-D DEM and to perform the parametric study. Basic simulation result obtained is the DOP on the thick target. Convergence study to get the optimal number of discrete elements is performed and the comparison of DOP obtained from 1-D DEM with 1-D FDM and experimental results of Woodward (1982) are done 
in this paper. Parametric study is conducted by observing the DOP with respect to change in impact velocity of penetrator. Details of simulations results are as shown in subsections below.

\subsection{Comparison of 1-D DEM results with 1-D FDM and experimental values}

Figure 7 is the comparison of DOP of the 1-D DEM results with FDM results for different impact velocities. In order to compare with 1-D FDM results, same number of elements is used as in FDM. Thus in 1-D DEM, 10 elements are used for penetrator and 20 elements are used for target to compare with 1-D FDM result of Woodward (1982). The trend shown in figure 7 is a nonlinear variation of DOP with respect to impact velocity. It is clear from the figure 7 that both 1-D DEM and FDM results are matching well for all impact velocities.

\subsection{Convergence study using 1-D DEM}

Several mesh sizes were simulated to study the convergence of the simulations with mesh sizes (figure 8). Combinations of target and penetrator sizes that were studied include: (a) 10 elements on penetrator and 20 elements on target, (b) 20 elements on penetrator and 40 elements on target, (c) 30 elements on penetrator and 60 elements on target, (d) 40 elements on penetrator and 80 elements on target and (e) 50 elements on penetrator and 100 elements on target. It is seen that as the elements increase from combination (c) to (e) the variation in DOP is less than $0.5 \mathrm{~mm}$. Thus 40-80 element combination is taken as the optimal element size and is used for further discrete element analysis.

\subsection{Variation of DOP with respect to change in border stress}

The border stress is the parameter which takes care of boundary effects in the target element. It is observed from figure 9 that as the constant parameter ' $C$ ' in the border stress varies, the DOP

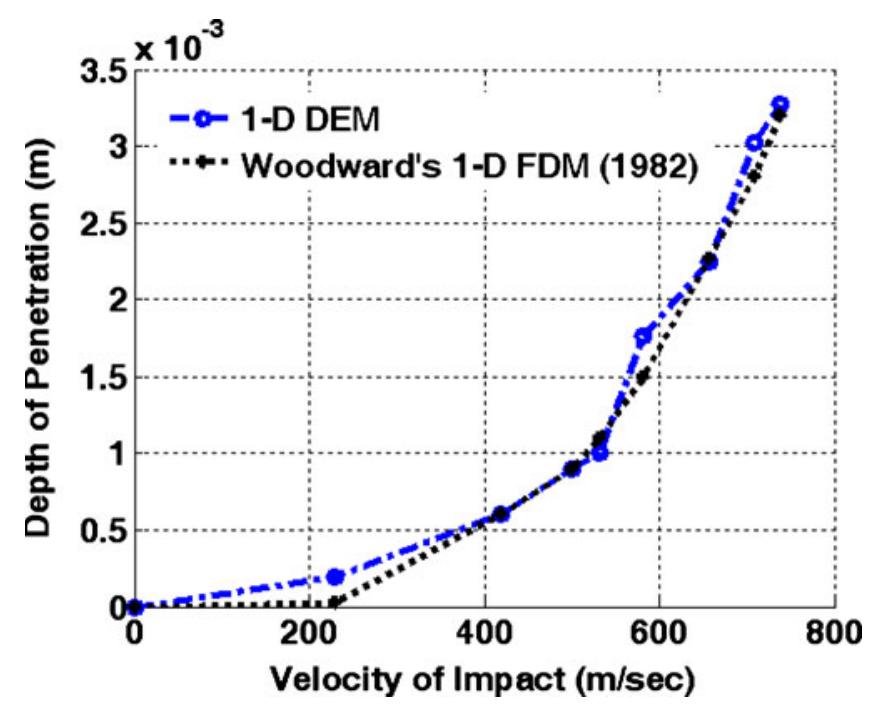

Figure 7. Comparison of 1-D DEM and 1-D FDM results (10-20 element combination). 


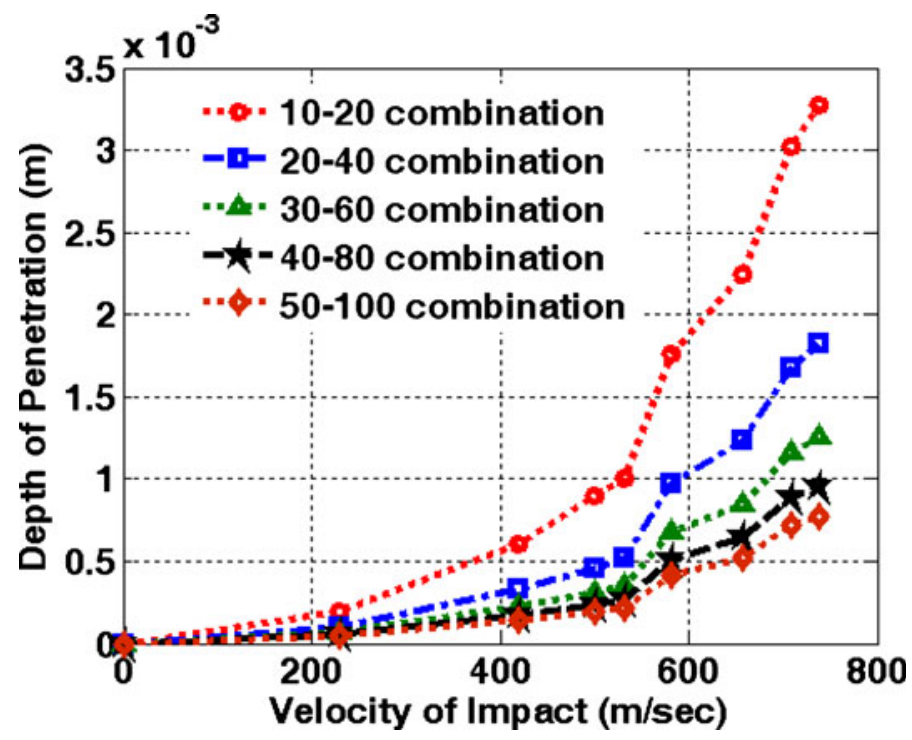

Figure 8. Converged study for ballistic impact using 1-D DEM formulation.

varies with respect to change in velocity of impact marginally. Thus it is observed that border stress constant is not an important parameter which governs the DOP. 1-D DEM simulations are thus done using $\mathrm{C}=0.577$ which is the same value used by Woodward (1982) for 1-D FDM. C is the constant from distortion energy theory of failure.

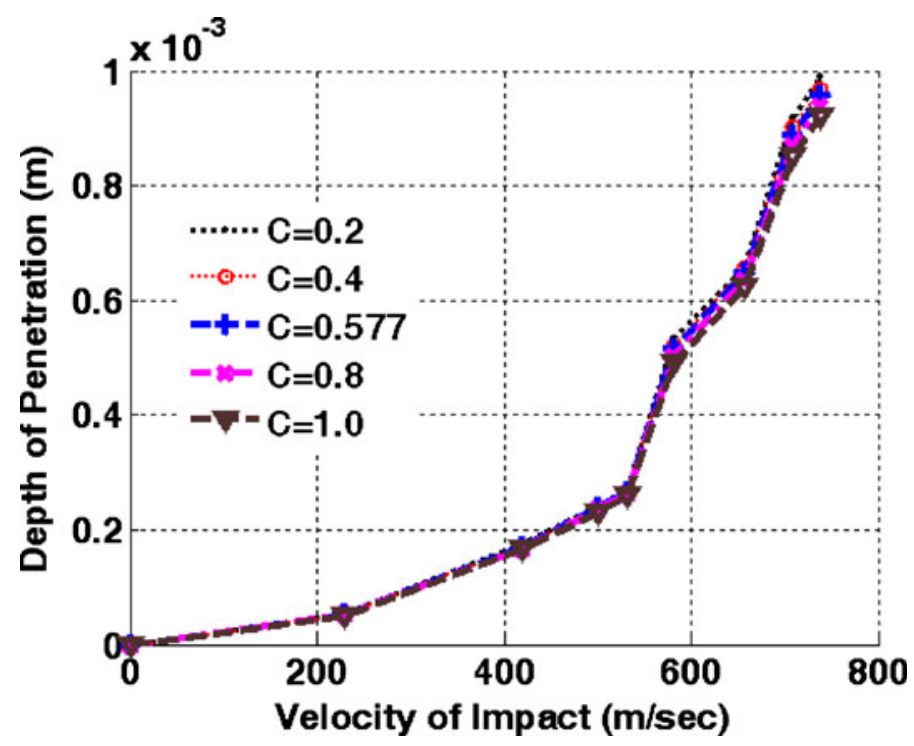

Figure 9. Variation of DOP with change in border stress for the converged elements (40-80 element combination). 


\subsection{Variation of penetration depth with impact velocity}

During ballistic impact, the penetrator may impact the target with different values of initial velocity. In order to study these effects, a parametric study is conducted to observe the variation of penetration depth with velocity of impact. Figure 10 shows the variation of DOP with respect to the velocity of impact, for the choice of material for penetrator and target as indicated in table 1 . Figure 10 indicates that for each value of initial impact velocity, the DOP of the penetrator tip increases nonlinearly with time and reaches a saturation value after a specified time. The penetrator tip stops moving at this saturation depth. The saturation DOP is the maximum value for the curve. It is observed that the saturation DOP increases with impact velocity. Further, the time for saturation also increases with impact velocity.

\subsection{Comparison of 1-D DEM results with experimental values}

Woodward (1982) had reported experimental measurements of DOP with impact velocity. The DOP was measured by Woodward (1982) using the X-ray diffraction technique and only one value was measured and reported for each impact velocity. Figure 11 shows a comparison of DOP predictions from the converged 1-D DEM results with the experimental results reported by Woodward (1982) for different impact velocities.

The trend shown in experimental and DEM predictions are nonlinear variation of DOP with respect to impact velocity. For lower velocity both DEM predictions and experimental observations are matching well with each other. For higher velocities, there is a discrepancy between the DEM results and experimental results. This mismatch can be due to any of the following reasons.

(i) The measurements reported by Woodward (1982) are not accurate and need to be verified with at least two or more observations at each impact velocity.

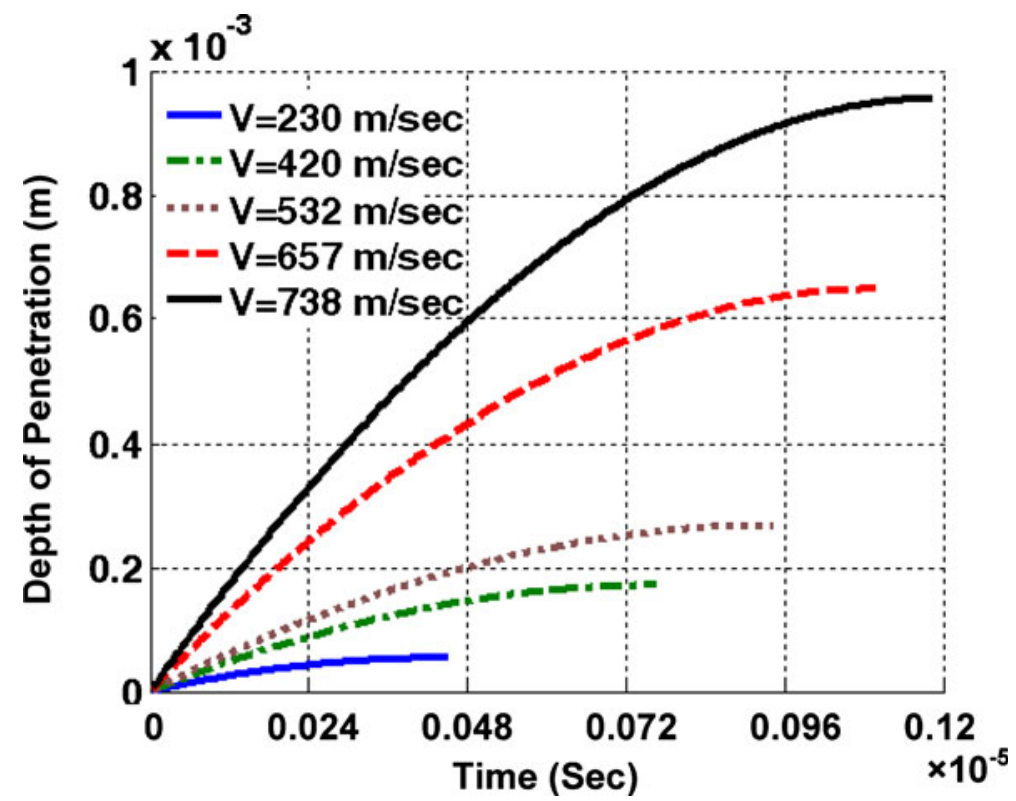

Figure 10. Depth of penetration calculated using 1-D DEM for different impact velocities. 


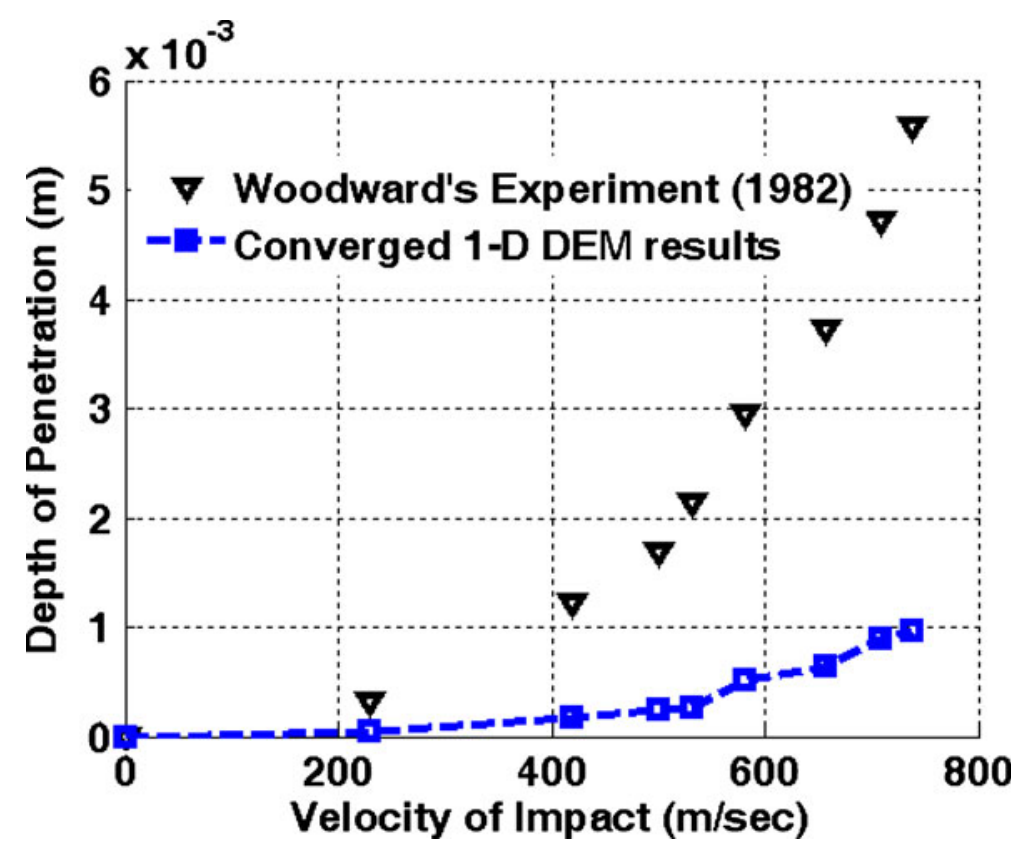

Figure 11. Comparison of converged 1-D DEM results with experimental results.

(ii) Damping effects may be significant at higher velocities and hence may affect the predicted depths of penetrations. Incorporation of damping effects is outside the scope of the current work and will be considered in our future work.

\section{Conclusions}

The basic motivation to write this paper was to prove that for getting DOP in ballistic impact, 1-D numerical simulation is sufficient and no need to go for 2-D or 3-D simulations which are computationally expensive. This paper deals mainly with the application of 1-D DEM for ballistic impact problem consisting of a flat end copper penetrator penetrating into a thick aluminum alloy target. The deformation and contact forces at all intersection points of discrete elements are obtained using 1-D DEM. From the parametric study it is found that velocity of impact has direct effect on DOP. A convergence study to obtain optimal number of discrete elements is done in this paper. Comparison of converged discrete element results with the experimental results (Woodward 1982) is plotted. Even if experimental results are not matching with DEM for higher velocities, DEM results are matching with FDM results (Woodward 1982) for all velocities. In literature, 1-D ballistic impact analytical models are available but very few 1-D numerical models are available for ballistic impact. The ability of a simple one-dimensional model using DEM in providing a good first order estimate of the DOP was demonstrated in this study. Also, the variation of the DOP with impact velocity gives useful results that will help in the design of impact systems. Using DEM, the separation of elements can be simulated easily compared to FDM or FEM which implies that DEM will give a realistic simulation of impact. This will be our future work. 


\section{List of symbols}

$\lambda$ Lame's parameter

$\mathrm{G}$ Bulk modulus

e Volume expansion

$\rho$ Density of the material

$u$ x-displacement in elastic media due to 3-D wave propagation

$v$ y-displacement in elastic media due to 3-D wave propagation

$w$ z-displacement in elastic media due to $3-\mathrm{D}$ wave propagation

$\sigma$ Stress on elastic rod

$\varepsilon$ Strain on elastic rod

$E$ Young's modulus of rod

$\Delta u$ Displacement of spring

$F$ Force on the discrete element mass

$A$ Cross sectional area of the rod

$l$ Length of discrete element

$K_{n}$ Normal spring stiffness between each discrete element

$K_{t}$ Normal spring stiffness between each target element

$K_{p}$ Normal spring stiffness between each penetrator element

$l_{p}$ Length of a penetrator discrete element

$l_{t}$ Length of a target discrete element

$\mathrm{V}_{t 1, t}$ Velocity of target element at the impact end

$\mathrm{V}_{p 1, t}$ Velocity of penetrator element at the impact end

$\mathrm{V}_{0}$ Initial velocity of penetrator

$\Delta t$ Time step in discrete element simulation

$u_{t 1, t}$ Displacement of the target element at the impact end

$V_{t n t, t}$ Velocity of target element at the non-impact end

$u_{t n t, t}$ Displacement of the target element at the non-impact end

$V_{p 1, t}$ Velocity of penetrator element at the impact end

$u_{p 1, t}$ Displacement of the penetrator element at the impact end

$F_{y}$ Shear force at the boundary of discrete element

$F_{I}$ Inertial force on the mass

$m_{t}$ Mass of target element

$\Delta t_{c}$ Critical time step of explicit integration

$\mathrm{m}_{\min }$ Minimum mass of the discrete element

$K_{\max }$ Maximum contact stiffness of the discrete element

$K_{t}$ Contact stiffness of target

$K_{p}$ Contact stiffness of penetrator

$\mathrm{F}_{c t 1, t}$ Contact force at impact end of target discrete element

$\mathrm{F}_{c p 1, t}$ Contact force at impact end of penetrator discrete element

$\mathrm{F}_{p 1, t}$ Total force at impact end of penetrator discrete element

$\mathrm{F}_{t 1, t}$ Total force at impact end of target discrete element

$C$ Constant calculated from distortional energy theory for failure

$d$ Diameter of penetrator

$h$ Length of target discrete element

$\sigma_{f}$ Flow stress of the element

$V(t)$ Velocity of discrete element as a function of time

$n$ Total number of time steps 
$T$ Total time for simulation

$\mathrm{m}_{p}$ Mass of penetrator discrete element

$\mathrm{m}_{t}$ Mass of target discrete element

$\mathrm{V}_{p 1,1}$ Velocity of 1 st penetrator element at 1 st time step

$\mathrm{X}_{p 1,1}$ Distance travelled by 1 st penetrator element after 1st time step

$\mathrm{V}_{p 1,2}$ Velocity of 1 st penetrator element at 2 nd time step

$\mathrm{X}_{p 1,2}$ Distance travelled by 1 st penetrator element after 2nd time step

$P$ Depth of penetration

\section{References}

An B, Tannant D D 2007 Discrete Element Method contact model for dynamic simulation of inelastic rock impact. Computers and Geosciences 33: 513-521

Anderson C E Jr, Littlefield D, Walker J D 1993 Long-rod penetration, Target resistance and Hypervelocity impact. Int. J. Impact Eng. 14: 1-12

Awerbuch J, Bodner S R 1974 Analysis of the mechanics of perforation of projectiles in metallic plates. Int. J. Solids Struct. 10: 671-684

Bathe K J 1996 Finite element procedures (India: Prentice Hall) 2nd Edition: 770-773

Ben-Dor G, Dubinsky A, Elperin T 2005 Ballistic impact: Recent advances in analytical modelling of plate penetration dynamics-A review. ASME. 58: 355-371

Cundall P A, Strack O D L 1979 A discrete numerical model for granular assemblies. Geotechnique. 29: $47-65$

Forrestal M J, Tzou D Y, Askari E, Longcope D B 1995 Penetration into Ductile Metal Targets with Rigid Spherical-Nose Rods. Int. J. Impact. Eng. 16: 699-710

Gailly B A, Espinosa H D 2002 Modelling of failure mode transition in ballistic penetration with a continuum model describing micro cracking and flow of pulverized media. Int. J. Numer. Meth. Eng. 54: 365-398

Hart R, Cundall P A, Lemos J 1988 Formulation of a three-dimensional distinct element model -part 2. mechanical calculations for motion and interaction of a system composed of many polyhedral block. Int. J. Rock Mech. Min. Sci. and Geomech. 25: 117-125

Hu B, Eberhard P 2004 Simulation of longitudinal impact waves using time delayed systems. Trans. ASME. 126: 644-649

Kurtaran H, Buyuk M, Eskandarian A 2003 Ballistic impact simulation of GT model vehicle door using FEM. Theoret. Appl. Fracture Mech. 40: 113-121

Magnier S A, Donze F V 1998 Numerical simulation of impact using a Discrete Element Method. Mech. Cohes-Frict. Mater. 3: 257-276

Prochazka P P 2004 Application of Discrete Element Methods to Fracture Mechanics of Rock Bursts. Eng. Fract. Mech. 71: 601-618

Raje N, Sadeghi F, Rateick R G Jr., Hoeprich M R 2007 Evaluation of Stresses around Inclusions of Hertzian Contacts using the Discrete Element Method. J. Tribol. Trans. ASME. 129: 283-291

Ravid M, Bodner S R, Holcman I 1987 Analysis of very high speed impact. Int. J. Eng. Sci. 25: 473-482

Sitharam T G 2000 Numerical simulation of particulate material using discrete element modelling. Curr. Sci. $78: 7-10$

Stronge W J 2004 Impact mechanics (UK: Cambridge University Press) 1st ed: 146-162

Tavarez F A 2005 Discrete element method for modelling solid and particulate materials, Ph.D. Thesis, University of Wisconsin-Madison, U.S.A.

Tavarez F A, Plesha M E 2007 Discrete element method for modelling solid and particulate materials. Int. J. Numer. Meth. Eng. 70: 379-404 
Timoshenko S P and Goodier J N 1970 Theory of elasticity (Singapore: Mc Graw-Hill International Editions) 3rd ed: 485-487

Woodward R L 1982 Penetration of semi infinite metal targets by deforming projectiles. Int. J. Mech. Sci. 24: 73-87

Woodward R L 1996 Modelling geometrical and dimensional aspects of ballistic penetration of thick metal targets. Int. J. Impact Eng. 18: 369-381 